\title{
Automatic Electronic Cleansing in Computed Tomography Colonography Images using Domain Knowledge
}

\author{
KN Manjunath ${ }^{1}$, PC Siddalingaswamy ${ }^{2 *}$, GK Prabhu ${ }^{1}$
}

\begin{abstract}
Electronic cleansing is an image post processing technique in which the tagged colonic content is subtracted from colon using CTC images. There are post processing artefacts, like: 1) soft tissue degradation; 2) incomplete cleansing; 3) misclassification of polyp due to pseudo enhanced voxels; and 4) pseudo soft tissue structures. The objective of the study was to subtract the tagged colonic content without losing the soft tissue structures. This paper proposes a novel adaptive method to solve the first three problems using a multi-step algorithm. It uses a new edge model-based method which involves colon segmentation, priori information of Hounsfield units (HU) of different colonic contents at specific tube voltages, subtracting the tagging materials, restoring the soft tissue structures based on selective $\mathrm{HU}$, removing boundary between air-contrast, and applying a filter to clean minute particles due to improperly tagged endoluminal fluids which appear as noise. The main finding of the study was submerged soft tissue structures were absolutely preserved and the pseudo enhanced intensities were corrected without any artifact. The method was implemented with multithreading for parallel processing in a high performance computer. The technique was applied on a fecal tagged dataset (30 patients) where the tagging agent was not completely removed from colon. The results were then qualitatively validated by radiologists for any image processing artifacts.
\end{abstract}

Keywords: Oral contrast - Colon segmentation - Hounsfield unit - Domain knowledge - Electronic cleansing

Asian Pac J Cancer Prev, 16, (18), 8351-8358

\section{Introduction}

Computed tomography colonography (CTC) is a medical imaging and diagnosis procedure for finding the colon polyps through image processing application using primary 2D Multi Planar Reformatted (MPR) and 3D volume rendered images (Rakesh, 2011). The endoluminal fluid and stool which obscure the polyp (Figure 1a) are the major cause of error in polyp diagnosis as the stool mimics polyp (Poullos et al., 2010). Patient is administered with oral contrast through a procedure called fecal tagging in which the stool shows homogenous attenuation coefficient (AC) when it absorbs the contrast (Figure 1b, d-e) whereas the soft tissue does not absorb (Tian, 2015) and their AC is unaltered (Figure 1b,e). This helps the Radiologist to distinguish polyp from tagged fecal matter. Clinical cases of fecal tagging in CTC are illustrated in Figure 1.

Electronic cleansing (EC) or virtual cleansing is an image post processing technique in CTC where the tagged colonic content is subtracted from colon without loss of soft tissue details. When contrast still remains in colon (Figure $1 \mathrm{~b}-\mathrm{c}$ ), it poses a great challenge in processing (Iinuma et al., 2008; Poullos et al., 2010) and leads to post processing artefacts. The consequences are, soft tissue structure erosion due to excessive processing, incomplete cleansing (IC) due to improper filtering (Cai et al., 2010; Lee et al., 2014) and pseudo soft tissue structures due to partial volume effect (PVE). IC mainly occurs in low dose CT scanning or medium dose bowel preparation which results in non-homogenous AC and demands more post processing. It leads to incomplete cleansing near air - contrast boundary (Figure 1b). Thick slices during CT image acquisition results in PVE where a voxel represents the information of more than one material. Incomplete cleansing may lead to wrong measurements and diagnosis (Manjunath, 2014). The evolution of engineering solutions published so far by others in solving these problems and the inference from their work are discussed below.

Literature review: EC methods are mainly classified in to statistical methods and edge model based methods (Lee et al., 2014). The first group classifies the voxel by its local features using Markov Random fields, expectation maximization and maximum a posteriori EM methods. The second group deals with precisely defining the type of transition between different colonic materials. Edge based methods discussed were, gradient based edge detection, arithmetic operation using vertical vector motion flow technique, voxel classification, material transition and local roughness analysis. Morphological and convolution based processing was discussed by Zalis et al (2004). The 


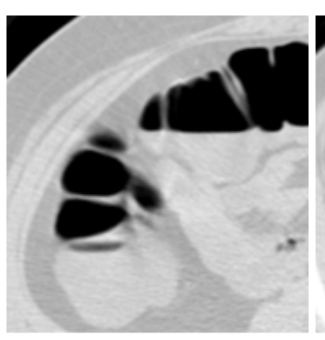

(a)

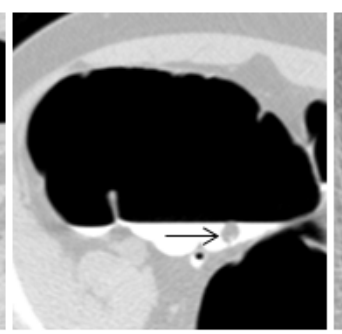

(b)

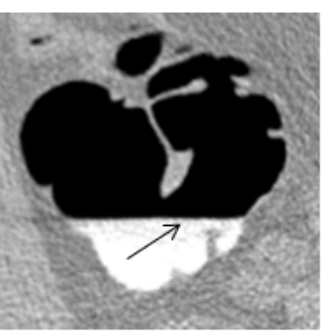

(c)

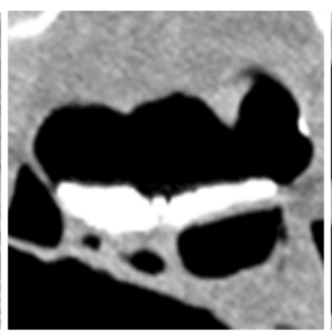

(d)

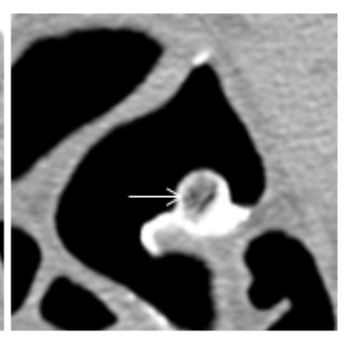

(e)

Figure 1. Generally Observed Fecal Tagging Cases in CTC. Viewed with window values W=1400, C=-350: a) Untagged fluids showing same HU for soft tissues and fecal matter where it is impossible to distinguish, b) Full dose colon preparation which shows a submerged polyp and high homogeneous HU for contrast, c) Medium dose colon preparation which shows heterogeneous HU near air-contrast boundary, d) Homogenous HU of tagged fecal material, and e) Tagging agent removed completely and the polyp surrounded by tagged fecal material

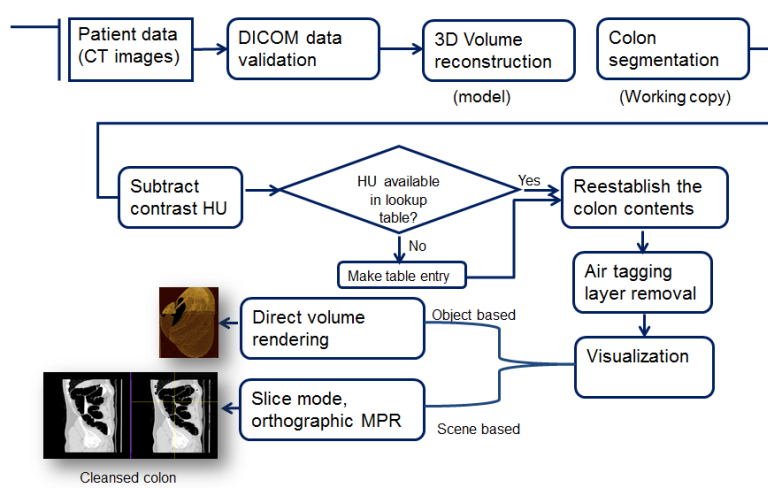

Figure 2. The Block Diagram of the Proposed Method

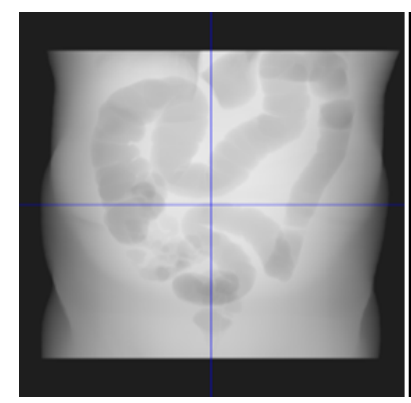

(a)

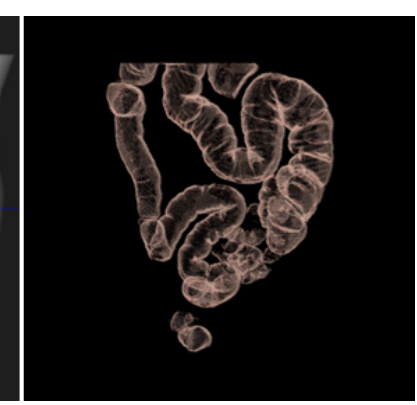

(b)
Figure 3. The Colon Visualization in Anterior to Posterior (AP) Direction. a) 2D Digitally Reconstructed Radiograph from original 3D volume (S) visualized with $\mathrm{W}=$ $573, \mathrm{C}=-389$, and b) the segmented colon (S0) visualized through direct volume rendering

evaluation of the method was limited to Phantom dataset. Wang et al. (2006) have discussed EC technique based on voxel classification. They infer that understating the HU range of various colonic contents can lead to better EC results without any artefact. Yamamoto et al (2009), have implemented EC with arithmetic operation using vertical vector motion flow technique. The limitation was, partial volume effect and uneven tagged residue was not removed properly and also the normal anatomical structure was eroded. Reduction of artefact near air-tagging boundary was discussed by Serlie et al. (2010) using Scale invariant Gaussian derivatives. It was applicable only for fully prepared colon and homogeneously tagged areas. The recent study using dual energy CT (Cai et al., 2013) with two different photon energies to identify two different materials was discussed. The technique was computationally expensive and dual energy CT scan lead to more radiation exposure to the patient. This was more when compared to the mean effective dose studied by a meta-analysis conducted by Chu et al. (2011). 3D scale based PE correction in order to address the misclassification of pseudo enhanced soft tissues and tagged material was discussed by Zhang et al. (2014). Lee has discussed on three material transitions using triple arc projection technique to remove the artefacts in EC. Their method mainly worked for homogenously tagged regions. The structure analysis scheme using morphological information instead of thresholding to differentiate the submerged soft tissues was discussed by Cai et al (2010). They used the combination of material transition, local roughness analysis, mosaic decomposition and erosion for removing PVE. Recent research (Serli et al., 2010; Cai et al., 2013; Lee et al., 2014) has focused on considering the material composition and the air-tagging boundary which has shown promising results to certain extent.

Many papers have discussed processing the images globally with specific threshold value and the CTC images acquired at particular $\mathrm{kVp}$ (tube voltage, a CT image acquisition parameter). We did not find any adaptive method which works with images acquired with different $\mathrm{kVp}$. The inference from the existing work is, an improved method of EC is needed which should ideally work in all different conditions as shown in Figure 1. The objective of the study is to clean the fecal tagging agent and to remove the air-contrast boundary without losing the tissue details. And achieving the same for images acquired at different $\mathrm{kVp}$. The following sections reveal materials \& methods, methodology of proposed technique, results, discussion, validation and conclusion.

\section{Materials and Methods}

This was a retrospective study and the anonymized clinical data (40 patients, 1000 images/patient) were obtained from National Cancer Institute, USA (NCI, 2015; Clark et al., 2013). The CTC protocol was followed as per ACRIN (Johnson et al., 2010) guidelines. Dataset with incomplete distention, motion artefacts and metal artifacts were discarded (5 patients) due to bad diagnostic quality of the images. Fecal tagging was carried out with Barium with medium dose and full dose colon preparation. Post fecal tagging, the tagging agent was partially removed 
in 30 patients and fully removed in 5 patients. Volume scanning of abdomen from diaphragm to pelvic region was performed with Multi detector CT (SIEMENS SOMATOM $^{\mathrm{TM}} 16,64$ slices and GE LightSpeed ${ }^{\mathrm{TM}} 16$ slices). The imaging details were - Section thickness: 1.0 - $2.5 \mathrm{~mm}, 100$ - $120 \mathrm{kVp}$, tube current: 60 - $300 \mathrm{~mA}$, Feet First Supine (FFS) and Prone (FFP) position scan, Image resolution 512 x 512, and the age of the patients were 40 to 80 . The data completeness was checked by validating the CTC images against the DICOM standards (DICOM 2012) for type 1 and type 2 attributes.

\section{Methodology}

The steps followed in the proposed approach are discussed in detail in this section. There are two tasks involved, a) cleaning of tagged colonic content from segmented colon and b) removal of air-contrast layer. The block diagram of the entire work is shown in Figure 2.

\section{Colon segmentation}

The patient data is validated initially against the DICOM (DICOM PS 3.3, 2012) standard and the 3D volume is reconstructed in $\mathrm{R}^{3}$ domain from the set of CT slices in $\mathrm{R}^{2}$ (Simone, 2010). As a first step, the volume is contrast corrected for adjusting the background details. The colon is extracted (Figure 3) with a hybrid semiautomatic segmentation method using domain knowledge of colon distention. In this approach, Canny edge detection is applied to detect the colon boundary. The parameters Low Threshold, High Threshold and Gaussian sigma $(\sigma)$ with values in the range 40-50, 100120 and 1.4 respectively resulted in accurate boundary detection between contrast-colon wall. Then colon blobs are identified using connected component labeling method and finally blobs diameter measuring $<2 \mathrm{~cm}$ are discarded using knowledge of colon distention grading (Pickhardt et al., 2014). Then the segmented blob region is filled with the original slice voxel intensities so that colon content is absolutely preserved without loss of tissue details. The relationship between original patient volume and the segmented colon is shown in Eq.1, where $\mathrm{S}$ is $\mathrm{V}_{\mathrm{g}}$ (given scene - patient volume), $\mathrm{S}_{0}$ is $\mathrm{V}_{\mathrm{b}}$ (binary scene - colon), and $\mathrm{f}, \mathrm{f}_{0}$ are the voxel intensities and $\mathrm{c}$ is the scene domain. $\mathrm{Vg}$ is gamma corrected (Eq. 2) for proper display where A is a constant and $\gamma$ is the gamma value of native display system.

$$
\begin{aligned}
& S=(c, f) \rightarrow S_{0}=\left(c, f_{0}\right) \\
& V_{\text {out }(i=0 . L-1)}=\mathrm{A} \cdot \mathrm{V}_{\text {in }} \gamma
\end{aligned}
$$

\section{Material composition}

The HU range is the key information in diagnosis of anatomies using Radiology images. In this application domain, internal makeup of the polyp should be homogenous soft tissue density in order to distinguish from tagged stool. The prior knowledge of material composition i.e. the HU of colonic contents (soft tissue, fecal tagging agent, carbon di-oxide, air and fat) at different $\mathrm{kVp}$ is the predominantly considered aspect in the proposed approach. The mass attenuation coefficient
Table 1. The HU Range of Colonic Materials at Different $k V p$ from Other Clinical Studies

\begin{tabular}{llll}
\hline & \multicolumn{3}{c}{ From clinical studies } \\
Tube voltage $(\mathrm{kV})$ & 80 & \multicolumn{1}{c}{100} & 120 \\
\hline Air & $-1000 \pm 10$ & $-1000 \pm 10$ & $-1000 \pm 10$ \\
Contrast & 144 & 138 & 130 \\
Soft tissue & $+62 \pm 5$ & $+58 \pm 5$ & $+54 \pm 5$ \\
Water & $0 \pm 5$ & $0 \pm 5$ & $0 \pm 5$ \\
Fat & $-152 \pm 10$ & $-111 \pm 10$ & $-89 \pm 10$ \\
$\mathrm{CO}_{2}$ & $-1000 \pm 25$ & $-1000 \pm 25$ & $-900 \pm 2$ \\
\hline
\end{tabular}

Table 2. The Observed HU Range of Different Materials at Different Tube Voltages from Secondary Data

\begin{tabular}{lll}
\hline & \multicolumn{2}{c}{ Observed HU range } \\
\cline { 2 - 3 } $\mathrm{kV}$ & \multicolumn{1}{c}{100} & \multicolumn{1}{c}{120} \\
\hline Soft tissue & +60 to +90 & +50 to +60 \\
Tagged fecal matter & +200 to +3000 & +200 to +2500 \\
Air-contrast boundary & -500 to +300 & -700 to +500 \\
Air & $-1000 \pm 15$ & $-1000 \pm 15$ \\
$\mathrm{CO}_{2}$ & $-1000 \pm 50$ & $-1000 \pm 50$ \\
\hline
\end{tabular}

$(\mu)$ of material varies due to variation in photon energies $(\mathrm{keV}) . \mu$ is the factor that results in tissue contrast in medical imaging. HU is calculated from $\mu$ using Eq. 3 . Recent study by Mah P et al., (2010) have discussed on calculation of HU from $\mu$ of various substances defined in NIST table. Calculated HU was cross verified with actual HU of CT images and they inferred that, both varies slightly which is acceptable during diagnosis. Based on empirical testing, $\mu_{\text {materials }}$ at different $\mathrm{keV}$ ( $1 \mathrm{keV}$ to $1 \mathrm{MeV}$ ) is described in NIST table (ICRU report 44, 2015; NIST 2015). With this as standard reference, theoretically HU can be calculated at different $\mathrm{keV}$ and can be used in EC. Eqn. 3 establishes the relationship between $\mathrm{keV}$ and the pixel intensity.

$$
\begin{aligned}
& 1000^{*}\left[\left(\mu_{\mathrm{t}}-\mu_{\mathrm{w}}\right) /\left(\mu_{\mathrm{w}}-\mu_{\text {air }}\right)\right] \\
& \text { ke } V \rightarrow \mu_{t}(x, y, z) \rightarrow \text { CTNumber }(x, y, z) \\
& \text { CT*m+Y int ercept } \quad[(\mathrm{HU}-\mathrm{P} 1) / \mathrm{w}]^{* 2^{\mathrm{i}-1}} \\
& \rightarrow \quad H U(x, y, z) \rightarrow f(x, y)
\end{aligned}
$$

Where, $\mu_{\mathrm{w}}, \mu_{\mathrm{t}}, \mu_{\text {air }}$ are the $\mathrm{AC}$ of water, tissue and air respectively, CT numbers of the material is calculated w.r.t $\mu_{\mathrm{w}}\left(\mu_{\text {air }}\right.$ is 0$), \mathrm{HU}$ is the voxel intensity calculated using rescale slope $\mathrm{m}$ and $\mathrm{y}$ intercept, $\mathrm{P}_{1}$ is the window left border, $W$ is window width, $\mathrm{i}$ is the radiometric resolution in $[0 . . L-1]$ and $\mathrm{f}(x, y)$ is the pixel intensity calculated after applying window center $(C)$ and window width (W). For example, if the AC considered for two materials from NIST table at $100 \mathrm{kVp}$ are $\mu_{\text {fat }}=0.194 \mathrm{~cm}^{-1}$ and $\mu_{\mathrm{w}}=0.222 \mathrm{~cm}^{-1}$, then as per Eq. 3, $\mathrm{HU}_{\text {fat }}=-126$ which is equivalent to the reported value $-111 \pm \mathrm{SD}$ in Table 1 . Normally in CTC scans, $80 \mathrm{kVp}-140 \mathrm{kVp}$ is used based on clinical task. Variation of $\mathrm{HU}$ as a function of $\mathrm{kVp}$ was discussed in a recent clinical study (Table 1); (Kalender, 2006; Huda, 2015). Their study involved normal size patients. Table 1 shows the HU observed within different Regions of interest (ROI).

Practically observed HU in our CTC dataset at two 


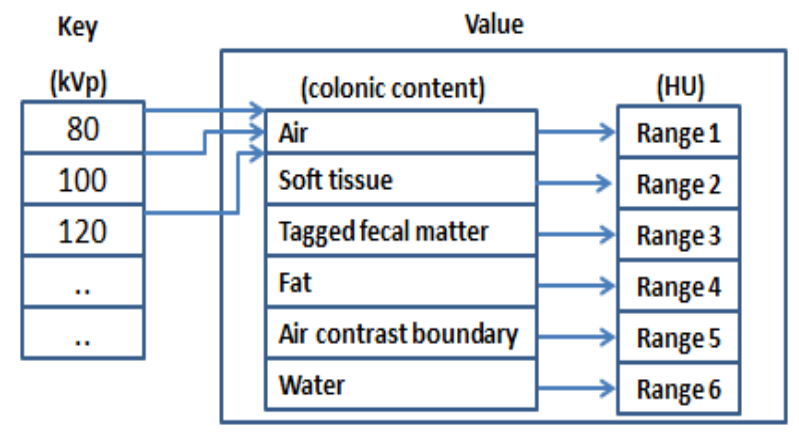

Figure 4. The Data Structure of the Lookup Table with Key Value Pair

different $\mathrm{kVp}$ are shown in Table 2. The common and key factor behind all the three cases discussed above i.e., w.r.t Eq.3, Table 1 and Table 2 is that the study was performed with normal size patient and there was no much variation of HU observed within ROI. Therefore we can rely on calculating $\mathrm{HU}$ of required materials directly from Eq. 3. By considering this as the ground information, a lookup table of $\mathrm{HU}$ has been created for various levels of $\mathrm{kVp}$ used in CTC scanning.

\section{Lookup table creation}

Based on the $\mathrm{HU}$ at particular $\mathrm{kVp}$ calculated using Eq. 3 or with reference to Table1/Table2, a lookup table is created with key as the $\mathrm{kVp}$ and the value as the colonic materials and their HU range. The table is a simple data structure defined as List $<\mathrm{kVp}$, List $<$ colonic contents, HU range $\gg$ (Figure 4). When the CT volume is loaded, the table is searched for the matching $k V p$ parameter of the dataset. If an entry is found, the HU range is picked, if not, a new entry is made based on the HU calculated with Eq. 3. Irrespective of images which are acquired at various $\mathrm{kVp}$, the range of $\mathrm{HU}$ is fetched adaptively from the lookup table for further steps of processing (restoring the soft tissue details). User does not have to define any parameter for EC.

\section{Electronic cleansing}

In the proposed EC method, the specific HU range of colonic content is post processed. Post colon segmentation (in working copy), the voxels $\mathrm{v}_{0}(x, y, z)$ are processed in the following order (Eq. 4). Initially voxels with intensity $\leq-$ $850 H U$ are explicitly set to $\min _{\mathrm{HU}}\left(\right.$ slice $_{\mathrm{i}}$ ) (condition 1 ). This removes the luminal noise. $\min _{\mathrm{HU}}\left(\right.$ slice $\left._{\mathrm{i}}\right)$ is the minimum HU in the current slice. The $\min _{\mathrm{HU}}$ is not same across all the CT slices and can vary slightly based on variation of tube current from superior to inferior direction (or vice versa) of patient scan. In the next step, $\mathrm{v}_{0}(x, y, z)$ $\geq+600 H U$ are set to $\min _{\mathrm{HU}}\left(\right.$ slice $_{\mathrm{i}}$ ) (condition 2 ). This removes the fecal tagging agent, tagged fecal matter as well as the boundary of submerged tissue structures. Lost soft tissue structure details have to be retained back. For this, HU of soft tissue and fat are fetched from lookup table using $3^{\text {rd }}$ condition and the voxel intensities of the result obtained from the previous step are replaced with these new values. This condition properly rectifies the pseudo enhanced (high density contrast overlaps with tissues) voxels near boundary between tissues and tagging agent and hence soft tissue erosion (problem 1 and 3 ) is prevented (Figure 6g). Only for soft tissues, the HU are elevated by around 80 , just to retain colon wall. Now colon is free from tagging agent. At this point the air tagging layer still persists (Figure 6f). It was observed that $H U_{\text {airtagginglayer }} \subseteq H U_{\text {contrast }}$ Since tagging agent and the tagged fecal matter shows high density, they are subtracted first based on condition 2 and then in the next step the airtagging layer is removed. Sequence of these conditions is important in the proposed method of EC.

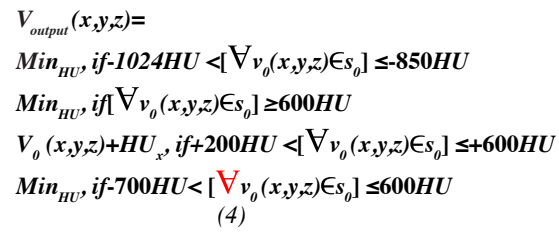

Where $\mathrm{HU}_{\mathrm{x}}$ is the soft tissue voxel range, $\mathrm{v}_{0}(\mathrm{x}, \mathrm{y}, \mathrm{z})$ is the voxel belonging to the segmented colon $\mathrm{S}_{0}$, and $\mathrm{V}_{\text {output }}$ is the result after EC. The HU range defined in Eq. 4 are the observed values at $120 \mathrm{kVp}$ from the secondary dataset. This range changes based on the $\mathrm{kVp}$ of CTC dataset and its associated HU defined in lookup table.

\section{Air-contrast layer removal}

When tagging agent still remains in colon it results a bowl shaped structure with high HU facing downwards when visualized on axial plane (Figure 5). Ideally just above the tagging agent, colon lumen should show $\mathrm{HU}_{\text {air }}$. But practically it does not as there are chances of floating minute fecal particles which appear as additive noise. Noise is more (due to heterogeneous HU) in case of medium dose and less (due to homogeneous HU) in case of full dose colon preparations. Heterogeneous HU is a major technical problem at present in removal of airtagging boundary. Theoretically a line can be assumed for this layer which shows specific voxel intensities (condition 4, Eq. 4) and practically it is true to some extent. By considering these two practical scenarios, voxels with same value of $\mathrm{v}_{0} . \mathrm{y}$ and $\mathrm{v}_{0}(x, y, z) \in H U_{\text {airtagging }}$ were searched. Collection of such voxels results in a thick line (Figure 5) formed due to surface of tagging agent. These lines have certain slope. With multiple dataset, empirically it was

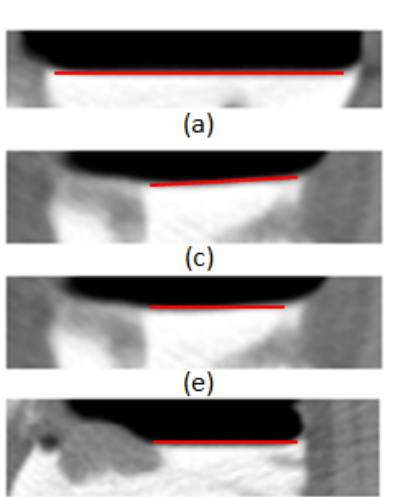

(g)

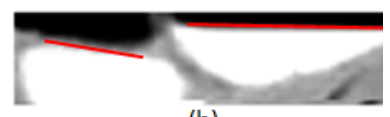

(b)

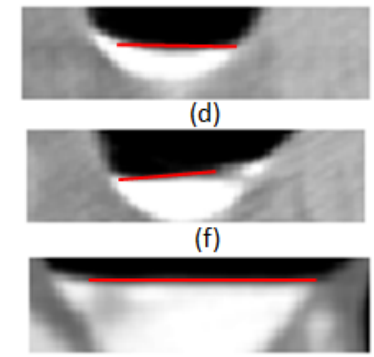

(h)
Figure 5. The Air-contrast Layer Representation. The red line shows the identified voxels with specific HU range near boundary. (a-h) lines with various slopes in the range $\mathrm{m}$ $=-0.5$ to 0.5 


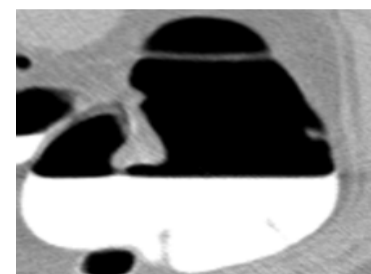

(a)

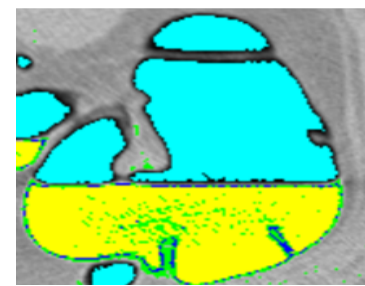

(e)

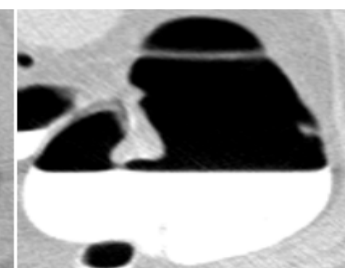

(b)

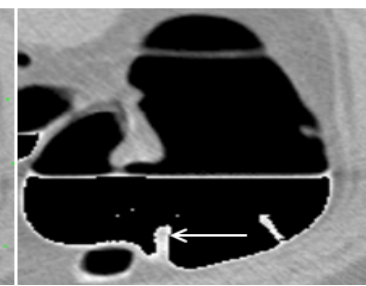

(f)

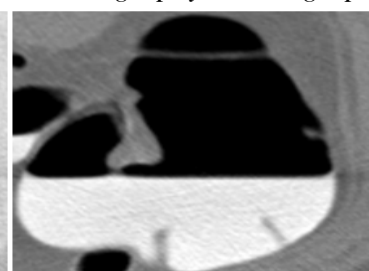

(c)

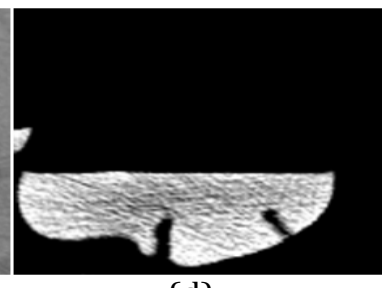

(d)

Figure 6. Results of Different Steps in the Proposed EC Method. (a-d) 2D view in transverse plane at different window values. a) $1500 \mathrm{~W},-200 \mathrm{C}$, b) $1400 \mathrm{~W},-350 \mathrm{C}$, c) $2000 \mathrm{~W}, 0 \mathrm{C}, \mathrm{d}$ ), custom $-400 \mathrm{~W}, 600 \mathrm{C}$, e) color coded regions, f) cleansed colon + air-contrast layer, g) air-contrast layer removed, h) removed noise through filtering

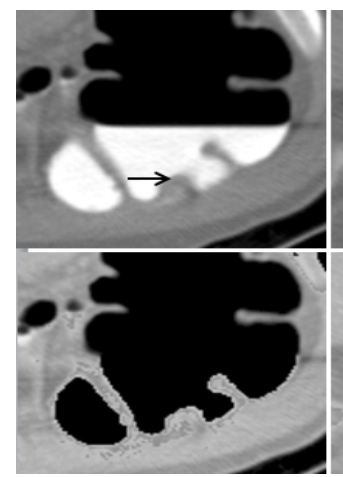

(a)

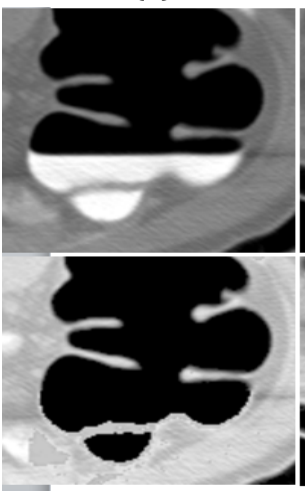

(f)

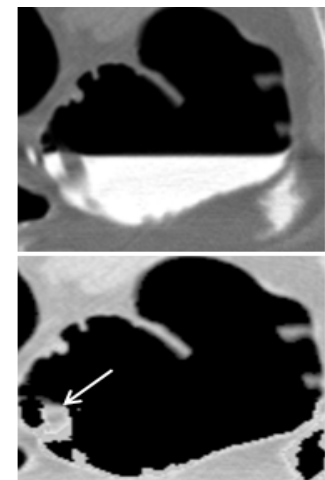

(k)

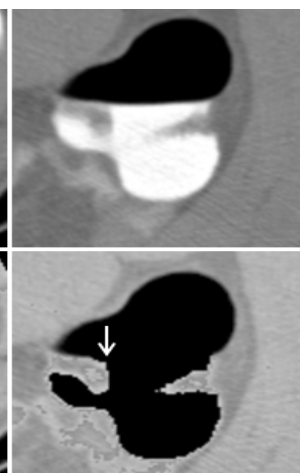

(b)

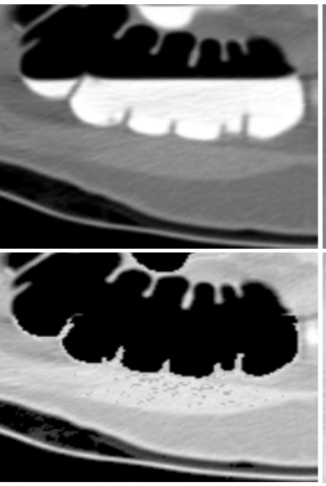

(g)

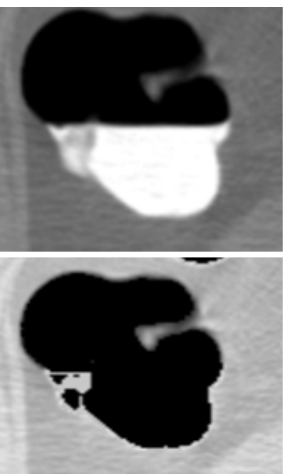

(1)

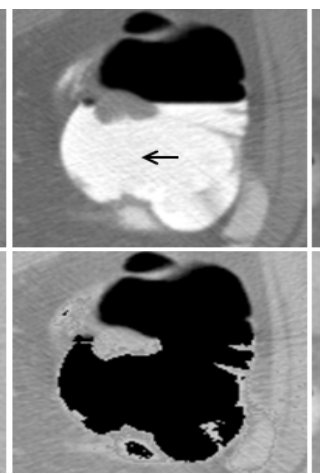

(c)

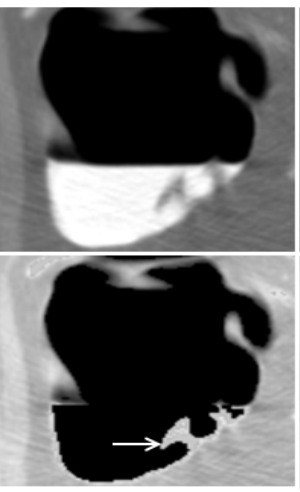

(h)

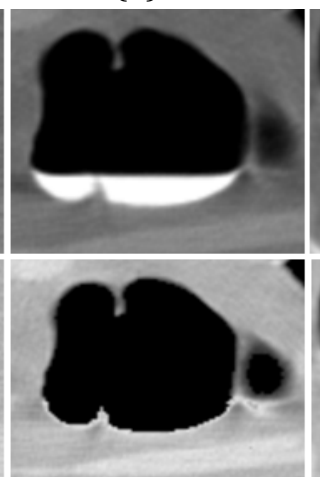

(m)

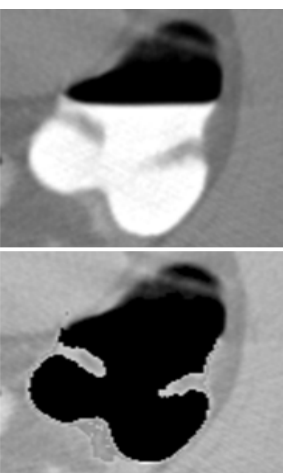

(d)

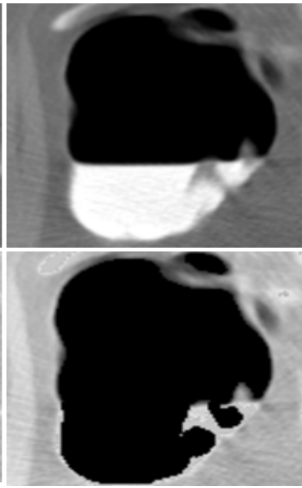

(i)

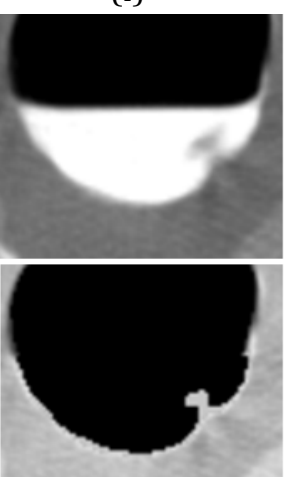

(n)

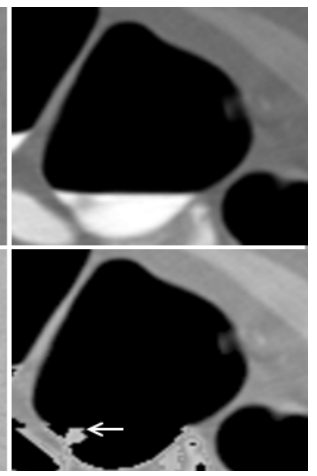

(e)

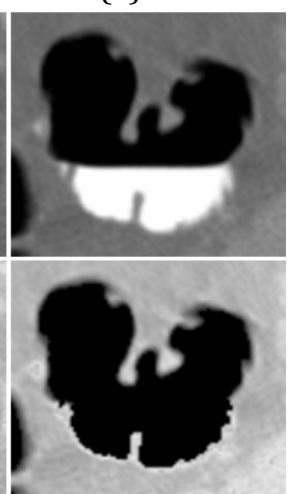

(j)

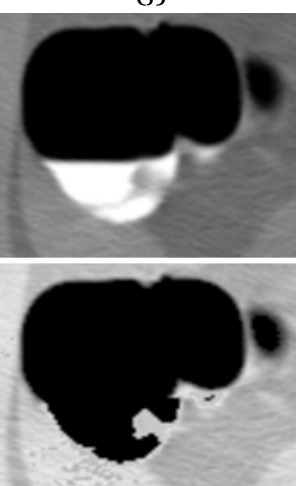

(o)

Figure 7. (a - e) Original Axial CT Axial Slices (row 1) and the Cleansed Images (row 2); (f - j) Original Axial CT Axial Slices (row 1) and the Cleansed Images (row 2); (k-o) Original Axial CT Axial Slices (row 1) and the cleansed images (row 2) 


\section{KN Manjunath et al}

found that these lines have slope in the range $-0.5 \leq \mathrm{m} \leq 0.5$. Searching of the additional voxels with the same logic is repeated $1-2 \mathrm{~mm}$ above this line. Finally all these identified voxels are set to $\min _{\mathrm{HU}}$. Both these layers are removed as per the $4^{\text {th }}$ condition (this range is the observed value from other literatures. This clears the air-contrast boundary completely excluding the adjacent soft tissue structures.

$$
g_{0}(x, y)=\text { Median }_{5 p o i n t}\left(f_{0}(x, y) \forall\left[f_{0}(x, y) \in s_{0}\right]\right)
$$

In the last step, the noise resulted due to improperly tagged endoluminal fluid is removed with median operator. Gaussian, adaptive and conservative smoothing methods were also tried. Since the images were processed to the maximum extent in the previous steps, all these methods resulted in soft tissue erosion. We found that simple 5 point median operator (Eq. 5) performed far better. The edges, especially the boundary of polyp and Haustras were not tampered. With this air-tagging boundary removal and incomplete cleansing (problem 2) is addressed.

\section{Results and Discussion}

EC was implemented on high performance workstation (Intel Xeon® CPU E52620 2.0GHz, NVIDIACUDA GPU, Windows 2012 Server 64 bit, 48GB RAM). A Prototype software has been developed with object oriented design and multithreading programming in Microsoft .NET framework 4.0. Microsoft volume rendering toolkit (Melancon 2010) has been used for 3D visualization. In our image pool, we have dataset acquired at 80, 100 and $120 \mathrm{KvP}$ with medium dose and full dose colon preparations. Scene based slice mode MPR with parallel navigation of original patient volume and the cleansed colon and object based volume rendering methods were used. We have not developed the endoluminal fly through navigation in our prototype for $3 \mathrm{D}$ visualization. But the same view of the colon has been produced by taking the clipping planes in arbitrary direction. Different window values reported in polyp analysis were $(2000 \mathrm{~W}, 0 \mathrm{~L})(\mathrm{Kim}$ and Pickhardt 2010), (1400W, -350L)(Poullos 2010) and (1500W, -150L)(Group investigators 2007; Slater et 1., 2006). We have used $1500 \mathrm{~W},-200 \mathrm{C}$.

Figure 6 illustrates the step by step result. The first row (a-d) shows the colon at three primary CTC window settings. As seen in Fig, it is difficult to find the submerged colonic contents as tagging agent obscures them. The W, C were manually selected towards lower value in HU scale to see the soft tissues (Figure 6d). For understanding purpose, different regions are color coded based on the HU range from the lookup table. Lumen air is represented by light gray, tagging agent by white, the air-tagging layer by black, and the soft tissues surrounded by improperly tagged endoluminal fluids by gray (Figure 6e). In the first step, the noise in colon lumen is removed and the tagging agent is subtracted. Slightly accentuated attenuation coefficients can be observed near the boundary of the Haustral fold (Figure 6f). This is due overlapping $\mathrm{HU}$ of tagging agent near tissue boundary. Figure $6 \mathrm{~g}$ shows the removal of air-contrast layer without soft tissue degradation and the accentuated HU near the boundary

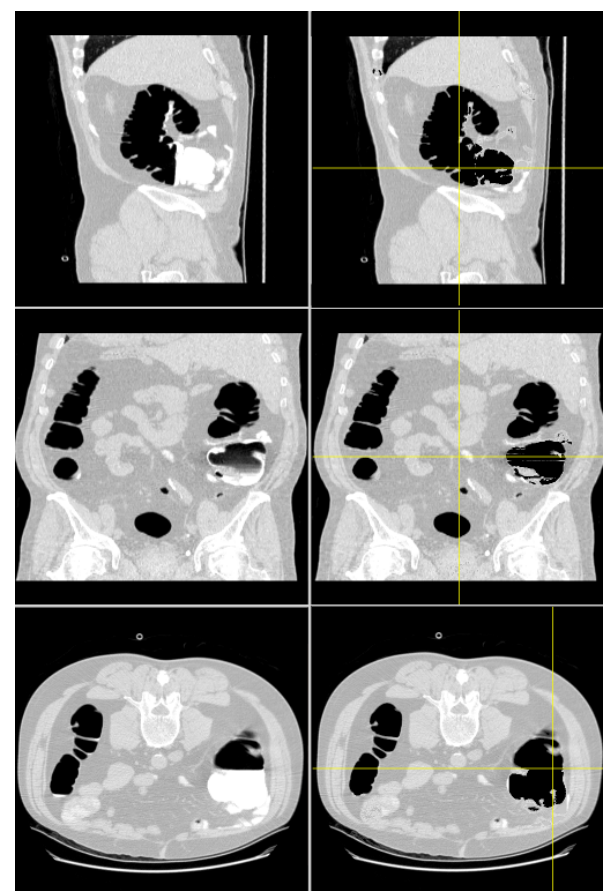

Figure 8. The Orthographic MPR Planes. column a) Original CT volume and column b) cleansed colon

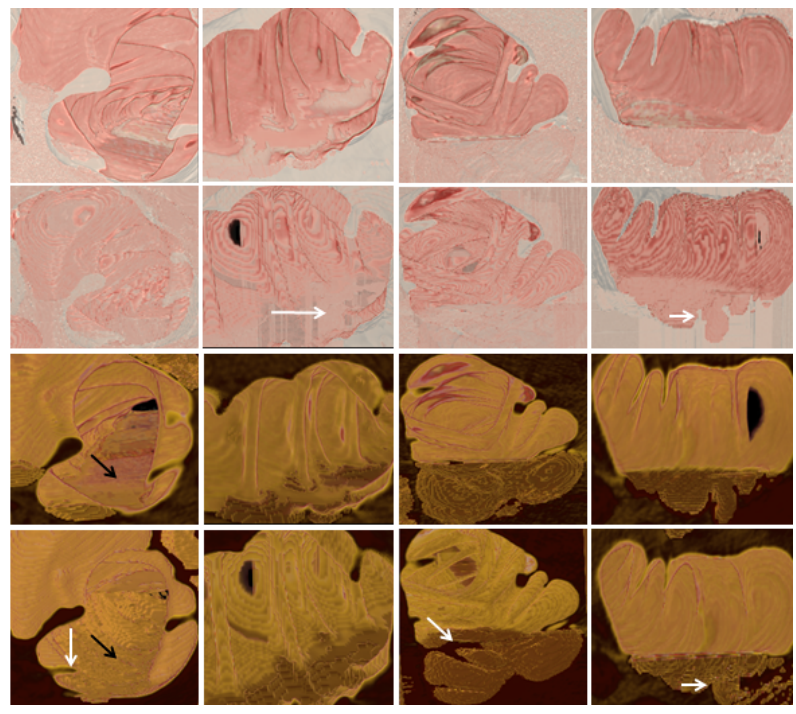

(a)

(b)

(c)

(d)

Figure 9. The Contrast Filled (row 1,3) and Cleansed Colon (row 2, 4) Visualized with Different Color Transfer Functions

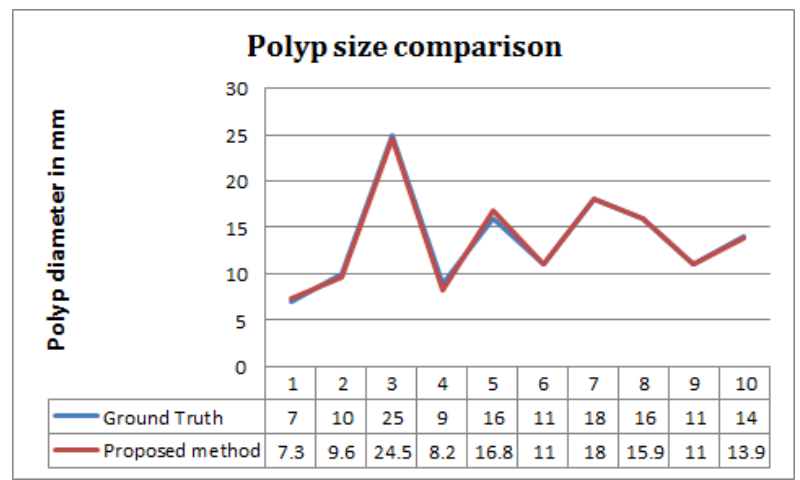

Figure 10. The Polyp Diameter Comparison after Electronic Cleansing 
is also reduced. This is the key finding in this approach.

The left out minute particle of improperly tagged fluids is removed with the help of median filtering with structure details unaltered (Figure 6h). The only noise encountered in colon is endoluminal noise which shows minor variations in $\mathrm{HU}$ irrespective of the $\mathrm{kVp}$ used. This was suppressed during the colon segmentation itself. Apart from this, improperly tagged fecal traces within tagging agent can appear as noise. Since both these noise possess very low HU, it is effectively handled in the proposed method. The results of different dataset are shown in Figure 7.

Partially and completely submerged soft tissue structures and the polyps are shown in Figure 7. Since the submerged contents are not properly visible in CTC window settings, the original axial slices are shown with colon window settings ( $\mathrm{W}=2000, \mathrm{C}=0$ ). The completely submerged haustra and the suspicious mass (arrow) can be seen in Figure 7a. The same structures are retained after EC with this approach. The Haustral fold which is adjacent to air-tagging layer and also which is partially submerged in tagging agent is retained without any artefact (white arrow in Figure 7b, h, k). The non - uniform HU due to improperly tagged endoluminal fluids (arrow) which appear as noise has successfully been removed in Figure 7c. The transition of $\mathrm{HU}_{\text {softissue }}$ towards the $\mathrm{HU}_{\text {contrast }}$ mainly resulted in soft tissue degradation in the earlier methods due to excessive processing (over filtering or processing of wrong HU). This limitation is properly handled in this proposed approach (condition 3, Eq. 4) and the results are illustrated in Figure 7a-e. A small sessile polyp which is submerged near the air-tagging boundary is completely preserved without any degradation (Figure 7e, 71) and also a polyp (Figure $7 \mathrm{~h}, \mathrm{n}$ ) which is completely submerged in tagging agent in the posterior direction has been retained after EC. None of the EC images has resulted in soft tissue degradation.

Figure 8 shows the entire patient volume visualized with MPR (sagittal, coronal and axial planes respectively). The reference line shows the cross sectional planes near lesion details in 3D volume. At this point the cleansed colon without any soft tissue structure degradation as well as properly removed air-tagging boundary can be observed (column b). The 3D volume rendered images are shown in Figure 9. First two and the last two rows are rendered with different color transfer functions. The first and the third row illustrate the original patient volume where the incompletely removed fecal tagging agent from colon shows bowl shaped structure with flat surface. This is removed completely (arrow, column b) after EC without loss of submerged tissue details. White arrow in Fig 9.a, 9.c and 9.d shows the Haustral fold which is retrieved back after EC. The same structures are not visible in the original volume where it is submerged in the tagging agent. The floating debris can be observed (black arrow, row 3 column 1) that appears as 1-2 mm layer of noise above the air-tagging layer. Air-tagging layer along with the floating debris has been removed without any side effects (black arrow, row 4, column 1).
The technique was applied on 30 CTC dataset. For results evaluation and comparison, we relied on the published results in different literatures which have done their research based on some of the commercially available EC solutions. EC results of the proposed method were comparable to the existing results. To know the accuracy, results were qualitatively validated. Supervised evaluation method was applied, which is widely used in medical image research. The results were demonstrated to three Radiologists and most two correlating readings were selected. Specifically the pseudo enhanced voxels near the soft tissue boundary and the retrieved polyp were checked. For Quantitative validation, after EC, randomly we measured the size of around 10 polyps from different dataset (Figure 10). The size was measured in 2D axial slice for the maximum diameter and compared with the previously published results (Johnson et al., 2008). The measurements were correlating and are illustrated in Fig 10. Consistency of the proposed method was checked by running EC for the same data set ' $n$ ' number of times $(\mathrm{n}=10)$, and no processing time difference was observed and hence method is reliable. The technique is efficient and practically viable; we were able to run this method for nearly 13-15 dataset (with 500 images/position scan) per hour without any breakdown of prototype.

In conclusion, we have proposed a new multistep image post processing method for electronic cleansing of colon which has given good results. Retrieving the submerged structures, avoiding the soft tissue degradation and correction of pseudo enhanced voxels are achieved based on the domain knowledge of HU of different materials. In the next step, incomplete cleansing issue is addressed using median filtering without tampering the tissue boundaries. The novelty in this approach is, irrespective of the $\mathrm{kVp}$ of CTC images, the HU are automatically calculated for different colonic materials and are added to the lookup table. Only the desired information is picked from the lookup table for further processing. We defend that practically in CTC clinical task when it is not possible to vary $\mathrm{kVp}$ for study purpose, the theoretical knowledge of HU calculation can be applied in EC. The main contribution in this work is retaining the finer details of partially and completely submerged soft tissues, correction of pseudo enhanced voxel intensities and removal of air-contrast layer without losing the adjacent tissue details. With this approach, images with medium and full dose colon preparation can be cleaned without any side effects. Overall it helps the Radiologist in analyzing the submerged polyp and any other lesions. Method was tested on dataset with various levels of contrast (partially filled, half-filled colon etc). The results were qualitatively checked by Radiologists for any post processing artefact and quantitatively checked the polyp size measurement with the ground truths. PVE is not addressed in this study as we did not have dataset with least submillimeter slice thickness. We evaluated this technique for the dataset with 80,100 and $120 \mathrm{kVp}$. It can be generalized for other voltage levels also.

\section{Acknowledgements}




\section{KN Manjunath et al}

We would like to thank National Cancer Institute, USA for providing the anonymized dataset required for the study purpose. We declare that we do not have any conflict of interest in this work including the CTC images from the secondary source. We extend our sincere thanks to Microsoft for volume rendering SDK. It is used only for academic research and not for any commercial purpose.

\section{References}

Ca W, Lee JG, Zalis ME, Yoshida M (2011). Mosaic decomposition: an electronic cleansing method for in homogeneously tagged regions in noncathartic CT colonography. IEEE Trans Med Imaging, 30, 559-74.

Ca W, Ki SH, Lee JG, Yoshida H (2013). Dual-Energy Electronic Cleansing for Fecal-Tagging CT Colonography. Radiographics, 33, 891-2.

Cai W, Yoshida H, Zalis ME et al. (2010). Electronic cleansing for noncathartic CT colonography: a structure-analysis scheme. Informatics in radiology, Radiographics, 30, 585602.

Chu LL, Weinstein S, Yee J (2011). Colorectal Cancer Screening in Women: An Underutilized Lifesaver. AJR Am J Roentgenol, 196, 303-10.

Clark K, Vendt B, Smith K, et al (2013). The Cancer Imaging Archive (TCIA): Maintaining and Operating a Public Information Repository. J Digital Imaging, 26, 1045-1057.

Group Investigators University College London (2007). Effect of directed training on reader performance for CT colonography: multicenter study. Radiol, 242, 152-61.

Huda W (2015). CT Radiation Exposure: An Overview. Current Radiology Reports. 1-16.

Iinuma G, Miyake M, Arai Y, et al (2008). CT colonographytowards applications for colorectal cancer screening. Asian Pac J Cancer Prev, 9, 833-40.

Johnson D (2010). ACRIN 6664 National CT colonography Trial", american college of radiology imaging network. 8-12.

Johnson CD, MMM, Chen MH et al (2008). Accuracy of CT colonography for detection of large adenomas and cancers. N Engl J Med, 359, 1207-17.

Kalender W (2006). X-ray computed tomography. Phys Med Biol, 51, 29-43.

Kim DH, Pickhardt PJ (2010). Radiologists should read CT colonography. Gastrointest Endosc Clin N Am, 20, 259-69.

Lee H, Lee J, Kim B, Kim SH, Shin YG (2014). Fast threematerial modeling with triple arch projection for electronic cleansing in CTC. IEEE Trans Biomed Eng, 61, 2102-11.

Li XB, Ye ZX (2015). Primary thyroid lymphoma: multi-slice computed tomography findings. Asian Pac J Cancer Prev. 16, 1135-8.

Mah P, Reeves TE, McDavid WD (2010). Deriving Hounsfield units using grey levels in cone beam computed tomography. Dentomaxillofac Radiol, 39, 323-35.

Manjunath KN, Swamy PC, Prabhu GK (2014). Feasibility of computed tomography colonography as a diagnostic procedure in colon cancer screening in India. Asian Pac J Cancer Prev, 15, 5111-6.

Melancon G, Munzer T, Weikopf D (2010). Volume rendering on server GPUs for enterprise scale medical applications. In Eurographics/IEEE-VGTC symposium on Visualization. $1-10$.

National Cancer Institute (2015). [Online]: public. cancerimagingarchive.net/ncia/login.jsf.

National Institute of Standards and Technology (2015, ) [Online]: http://physics.nist.gov/PhysRefData/XrayMassCoef/tab4 . html.
Pickhardt PJ, Bakke J, Kuo J, et al (2014). Volumetric analysis of colonic distention according to patient position at CT colonography: diagnostic value of the right lateral decubitus series. AJR Am J Roentgenol, 203, 623-8.

Poullos PD, Beaulieu CF (2010). Current techniques in the performance, interpretation, and reporting of CT colonography. Gastrointest Endosc Clin N Am, 20, 169 - 92.

Rakesh S (2011). Recent advances in intestinal imaging. Indian $J$ Radiol Imaging, 21, 170-5.

Report 44 (2015). Tissue substitutes in radiation dosimetry and measurement. international commission of radiation units and measurement.

Serli IW, Vos FM, Truyen R, et al (2010). Electronic cleansing for computed tomography (CT) colonography using a scale-invariant three-material model. IEEE Trans Biomed Eng, 57, 1306-17.

Simone P, Faccioli N, Zaccarella A et al (2010). The diagnostic contribution of CT volumetric rendering techniques in routine practice. Indian J Radiol Imaging, 20, 92-7.

Slater A, Taylor SA, Burling D, et al (2006). Colonic polyps: effect of attenuation of tagged fluid and viewing window on conspicuity and measurement-in vitro experiment with porcine colonic specimen. Radiology. 240, 101-9.

The DICOM chapter 3 (2012). PS 3.3. NEMA, USA, 390 - 1174.

Tian SF, Liu AL, Wang HQ et al (2015). Virtual non-contrast computer tomography (CT) with spectral $\mathrm{CT}$ as an alternative to conventional unenhanced ct in the assessment of gastric cancer. Asian Pac J Cancer Prev, 16, 2521-6.

Wang Z, Li X, Li L, et al (2006). An improved electronic colon cleansing method for detection of polyps by virtual colonoscopy. In Conf Proc IEEE Eng Med Biol Soc, 6, 6512-5.

Yamamoto S, Iinuma G, Suzuki M, et al (2009). A simple image processing approach for electronic cleansing in computed tomographic colonography. Biomed Imaging Interv J, 5, 1-5.

Zhang H, Li L, Zhu H, et al. (2014). Integration of 3D scale-based pseudo-enhancement correction and partial volume image segmentation for improving electronic colon cleansing in CT colonography. J Xray Sci Technol, 22, 271-83.

Zalis ME, Perumpillichira J, Hahn PF (2004). Digital subtraction bowel cleansing for CT colonography using morphological and linear filtration methods. IEEE Trans Med Imaging. 23, 1335-43. 